\title{
AC 2011-2175: NEED ASSESSMENT FOR TA TRAINING: A SURVEY TO CAPTURE PARTICULAR NEEDS AT AN INSTITUTION
}

\section{Sohum Sohoni, Oklahoma State University}

Dr. Sohoni is an Assistant Professor in the School of Electrical and Computer Engineering at Oklahoma State University. He received his $\mathrm{PhD}$ in computer engineering from the University of Cincinnati in 2004 and his Bachelors in electrical engineering from COEP, Pune University in 1998. Dr. Sohoni's research interests are broadly in the area of computer architecture and performance analysis of computer systems. His primary field of research is the cache memory performance of memory-intensive applications. He has published in peer-reviewed conferences and journals such as ACM SIGMETRICS, IISWC, and the IEEE Transactions on Computers. His research is supported by the National Science Foundation and the Department of Justice through the Center for Telecommunications and Network Security at OSU. He has recently expanded his research interests to include engineering education, and has published his work at ASEE's national conference and ASEE's Midwest section conference. He advises several undergraduate, M.S., and PhD students.

\section{Donald P. French, Oklahoma State University}

Professor in the Department of Zoology, Coordinator of the Certificate Program in University Faculty Preparation, Coordinator of the Introductory Biology course and a Past-President of the Society for College Science Teachers. His research focus is on biology education including the use of inquiry and technology in the teaching introductory biology lecture and laboratories. He has published articles on the impact of teaching in reformed courses on graduate students.

\section{YoonJung Cho, Oklahoma State University}

Assistant professor in the School of Applied Health and Educational Psychology at Oklahoma State University. Her research is focused on students' achievement motivation and self-regulated learning process as well as teachers' motivation and its impact on instructional practices, both in traditional classroom setting and online instruction. She published articles on graduate teaching assistants' professional development as well as faculty development. 


\title{
Need Assessment for TA Training: A Survey to Capture Particular Needs at an Institution
}

\begin{abstract}
A widely-acknowledged factor inhibiting education engineering reform, interfering with learning, and reducing student retention is the inexperience and lack of pedagogical education of many instructors. This is particularly acute among graduate teaching assistants (GTAs), who often contribute the majority of contact hours within engineering courses and may gain little pedagogical content knowledge before they assume the role of primary instructor in graduate school or their first academic appointment. Often their preparation is little more than a syllabus and the textbook. An important first step in addressing this issue for engineering educators, whose responsibility is to supervise and mentor GTAs, is to identify the factors to be considered and assessed in developing an effective professional development program for GTAs.
\end{abstract}

We developed a need assessment survey to measure GTAs' perceived importance of various roles and responsibilities. Our results provide strong evidence that it is a valid and reliable measure. The participants rated the importance they placed on each of 24 GTA roles and responsibilities on a 5 point Likert scale. Analysis of the data included an exploratory factor analysis and reliability tests to ascertain the construct validity and reliability of the survey. Factor analysis was conducted with oblimin rotation with the 24 items of GTA roles. The eigenvaluegreater-than-one rule was initially used in combination with a scree test to determine the number of factors that would appropriately represent the concept of GTA roles and responsibilities. The results indicated a four-factor structure, accounting for approximately $54.03 \%$ of the total variance. Based on the four-factor structure that an exploratory factor analysis indicated, we conceptualized four categories of GTA roles and responsibilities; 1) clear communication, 2) student management, 3) preparation for feedback and assessment, and 4) course management /policy knowledge. To examine concurrent construct validity, we examined how our new survey is related to teacher motivation measure, one of the empirically validated measures.

Reliability coefficients with the sample of this study for the overall measure and for the four subscales of the survey ranged between 0.77 and 0.82 . The correlation analysis provided concurrent validity of the measure because the four factors were positively related to GTAs' intrinsic motivation to teach.

Although the survey showed favorable psychometric properties, further testing is warranted to confirm that the new measure of GTA's need assessment can be used as a reliable and valid tool across institutions. 


\section{Introduction}

Concerns about recruitment and retention of students in engineering disciplines have resulted in numerous calls for reform in engineering education ${ }^{[1-3]}$. Regardless of the chosen response to such calls, it is clear that quality education requires the presence of instructors who have learned to teach effectively. Unfortunately, because we often rely on "on-the-job" training, faculty become skilled at teaching after receiving their doctoral degrees and "practicing" on students. For this reason, institutions commonly establish teaching effectiveness centers dedicated to faculty development. Moreover, and of greater concern to us, much undergraduate teaching, especially during laboratories which may constitute $50 \%$ or more of the time that students are in the classroom, is performed by Graduate Teaching Assistants (GTAs) who may receive no training in pedagogy prior to their first teaching experience. Given the importance of instruction in determining students' willingness to pursue undergraduate degrees in engineering ${ }^{[4]}$ and the critical role the first year laboratory can play for students making the transition from high school to college ${ }^{[5]}$, the pedagogical knowledge and skills of GTAs becomes even more critical. Sadly, while one would predict that the inexperienced would be aware of their lack of skill and pedagogical knowledge and would seek assistance, this does not always appear to be the case. Perhaps this indicates that GTAs are performing at the expected level for their profession. Alternatively, this may indicate ignorance of expectations or lack of interest or motivation.

We conducted the research described here to ascertain, through surveys of faculty, GTAs, and students, what characteristics are considered to be most important in successful engineering GTAs and what are the perceptions of GTAs' competency levels. This study was undertaken in preparation for the development and implementation of a GTA training program, both to set the objectives of the program and to establish a baseline for measuring the impact of the program in the future. We tested psychometric properties of the measure by examining factor structure, reliabilities and concurrent construct validity. To examine concurrent construct validity, we examined how our new survey is related to teacher motivation measure, an empirically validated measure.

Results from an initial administration of the survey, and their impact on designing the TA Training program at OSU were presented in a prior publication ${ }^{[6]}$. An implication for the TA training program development from the current results is also provided in the Discussion section.

The rest of the paper is organized as follows. The next section, Background and Rationale, provides justification for TA training, insights from previous research on training GTAs, and common problems faced by GTAs. The Method section provides details on the survey participants, the components of the survey, teacher motivation measure, and procedures. The Results section provides results for the factor analysis and other reliability and validity tests. The 
Discussion section explains how the results provide evidence for the reliability and validity of the survey, and provides an example of how the results may be used to develop or improve a training program for GTAs.

\section{Background and Rationale}

It is logical to think that variations within courses (i.e. across sections or semesters) can affect students and programs. When engineering courses are taught by different faculty members and graduate teaching assistants using different teaching approaches, students' course satisfaction and learning outcomes vary considerably among courses. While oversight of course content often exists, students taking the same class under different instructors often have significantly different levels of conceptual understanding and skills gained from that course. This can impact subsequent courses that require pre-requisite knowledge. Variations in teaching quality among faculty can be addressed through professional development programs that introduce instructors to the best practices and latest developments in pedagogy and course design. However, although graduate teaching assistants play an important role in engineering student learning, many higher education institutions do not have a professional development program for GTAs in place ${ }^{[7]}$. Therefore, another solution to the variability in student understanding and satisfaction among sections or semesters might be to provide GTAs with the same professional development training, so labs and discussion sections are taught well and in a consistent manner. While extensive GTA training programs have their merits ${ }^{[8,9]}$, there exists the question about whether programs less costly in time or money could be sufficiently beneficial. To test this, one starting point would be with an evaluation of the expectations for GTAs and the degree to which they meet expectations without training.

As the nation tries to improve STEM education on many different fronts, work focusing on GTA training, must not be overlooked. At doctoral degree granting institutions, GTAs may contribute substantially to undergraduate education ${ }^{[10]}$. Many engineering courses have large lab components predominantly taught by GTAs. For a typical 3 credit-hour engineering class at our institution, faculty teach two 50-minute lectures, while less experienced GTAs teach two hours of lab per week. Because GTAs interact as much with students as do faculty, training in classroom management and pedagogy for these novice instructors may have greater impact on student learning and retention than similar efforts aimed at more experienced faculty, who may be set in their ways or may already be more accomplished instructors. A training program may reduce the negative impact of having GTAs who do not consider themselves teachers, or who are not aware of their roles and responsibilities. Because GTA selections are often influenced by advisors' needs for their research programs and which graduate students 'need' funding, irrespective of whether they are qualified or prepared to be GTAs, teaching quality among beginning GTAs can be extremely uneven. GTAs often receive no or little systematic training ${ }^{[11-}$ 14], and are unprepared to take on their teaching responsibilities due to lack of exposure to the best practices in teaching and classroom management ${ }^{[15,16]}$. At the institution under study, there 
exists little coordination of the selection and training of GTAs beyond requirements for international GTAs to pass an exam and receive some training on culture and communication, and safety training for all GTAs.

In science and engineering courses, there is a long history of using homework, labs, and discussion sections, which at universities with substantial graduate programs become the primary domain of the GTAs, as a means of providing students with the opportunities to practice and assess their conceptual understandings and higher order cognitive skills. Thus, GTAs become a substantial source of feedback and the primary contact for individualized instruction ${ }^{[17]}$. Laboratories provide opportunities for "hands-on" experience favored by concrete, transitional and sensory learners ${ }^{[18]}$. While laboratories help students learn to make data-driven decisions ${ }^{[19]}$, their primary goal in the minds of faculty is to afford students more time to learn concepts ${ }^{\text {[20] }}$. The laboratory environment and the influence of the GTA on that environment have been shown to play a major role in student retention in STEM disciplines ${ }^{[5]}$. Given the increased responsibilities of GTAs and their impact on student learning, preparing GTAs to be effective teachers is critical in not only retaining undergraduates and improving student learning and engagement, but also in retaining qualified college instructors. Many new engineering undergraduate students make decisions related to their future academic endeavors based on their perceptions of those GTAs. Moreover, the GTAs, if unprepared and untrained as teachers, will be more likely to experience frustration and failure ${ }^{[21]}$, which may affect their own decision to leave academia.

Even with training programs, new GTAs still consider college teaching a challenge ${ }^{[22]}$ when their pressing concerns in relation to teaching are not appropriately addressed in the GTA training program. Thus, identifying and incorporating concerns of GTAs into the development program is a key in enhancing the effectiveness of the training programs for GTAs. Fuller ${ }^{[23]}$ suggested that to ensure effective teacher development programs, it is critical to assess teacher concerns accurately. In addition, teacher training or professional development programs that do not reflect the needs and interests of participants are unlikely to motivate them, which in turn can result in the failure to attain the program's educational goals and objectives. This speaks directly to the importance of need assessment surveys designed to identify what motivates and concerns teachers in advance of developing training programs.

According to Sprague and Nyquist ${ }^{[24]}$, GTAs go through three stages of development in their role as a teacher, which include 'Senior Learners', 'Colleagues in Training', and 'Junior Colleagues'. Nyquist and Wulff ${ }^{[25]}$ suggest that faculty could use these developmental stages to determine how to approach their mentoring of GTAs in relation to teaching roles and assignments. GTAs in different stages of development are considered to have different concerns about teaching issues. Beginning GTAs are viewed as 'Senior Learners' because they have more expertise in the subject matter than most undergraduate students, but still identify themselves more with students 
rather than with faculty. These GTAs at the first stage of development are mainly concerned with how to survive and avoid making mistakes and thus tend to focus on issues like getting better evaluations from both students and supervisors. As GTAs gain more teaching experience, they become 'Colleagues in Training' and their concerns tend to switch to issues like teaching skills or teaching methods. Finally, more seasoned GTAs become 'Junior Colleagues' who consider themselves as fellow faculty and become concerned with the impact they may have on student learning and engagement. Most studies on teacher concerns have employed survey methods and most surveys were developed based on Fuller's ${ }^{[23]}$ model. Researchers often revised and extended survey items to reflect unique needs and characteristics of diverse teacher populations and teaching contexts. Mok $^{[26]}$ proposed that teacher concerns are context-specific and therefore different teacher populations in varying educational contexts may express different teaching concerns, which should be considered to address teaching concerns appropriately within GTA development programs.

An important first step in addressing this issue for engineering educators, whose responsibility is to supervise and mentor GTAs, is to identify the factors they will consider and assess in developing an effective professional development program for GTAs. Although it is very important to identify areas of focus for GTA training and development, there has been scant research on GTA needs for professional development. GTA need assessment will provide valuable information on how to best customize GTA training in a way to maximize its effectiveness and impact. As such, it is essential to have a well-developed need assessment tool to be able to identify important but under-prepared GTA roles and responsibilities. The present study focuses on developing a GTA need assessment survey and investigating its psychometric properties to provide empirical evidence that the survey is a valid and reliable tool that can be used to capture areas for GTA training.

\section{Method}

\section{Participants}

Invitations to participate in the online survey were sent out via email to all engineering graduate students $(\mathrm{N}=900)$ at the institution. Email lists were provided by the Office of Institutional Research, and invitations were sent so that the identity of each invitee was kept anonymous. We were interested in capturing the views of all graduate students (GTAs or not) in this project. However, for this study we used a subset of the participants who either currently work as GTAs or have done so in the past. Out of a total of 216 students who responded to the survey, 144 students met the above criteria. Of the 144 graduate teaching assistants, $85.6 \%$ were male, $10.0 \%$ were female, and $4.4 \%$ did not report. $66.3 \%$ were master's degree students and $33.7 \%$ were doctoral students. Although the proportion of female participants in our sample is significantly

smaller than that of male participants, it actually represents the male/female ratio $(81.5 \%$ male students, $18.5 \%$ female students) in the engineering student population of the participating 
institution. The GTAs spanned the entire teaching experience range of teaching assistants, from those who were first time GTAs to those who had taught up to 12 semesters. All results provided below are for these 144 survey respondents.

\section{Procedure}

The survey was administered using SurveyMonkey (www.surveymonkey.com) and consisted of six sections: information required for informed consent, Needs Assessment Survey Importance Ratings (24 items), Needs Assessment Survey Competence Ratings (24 items), Teacher Motivation Survey (15 items), demographic data (10 items) - personal characteristics and career plans, and demographic data (10 items) - employment specifics. Participants could skip any section or question. We offered participants the opportunity to add comments at the end of each opinion section.

\section{Need Assessment Survey}

The authors developed a need assessment survey to capture to what extent GTAs rate the importance of typical GTA roles and responsibilities such as providing feedback to students, grading student work in a fair and consistent way, and establishing a working relationship with students, etc. All roles and responsibilities chosen are provided in Table 1 of the results. The survey items were drawn from the literature or created by the authors. The instrument was developed to incorporate the following features:

- Relevant and applicable in GTA training settings

- Inclusive and comprehensive to capture GTAs' perceptions and needs for professional development in higher education settings

- Multi-dimensional (e.g., instructional practices, classroom management, engagement with students)

The participants were asked to rate the importance they placed on each of 24 GTA roles and responsibilities on a 5 point Likert scale with 1 representing 'Not at all important' and 5 representing 'Critically important' on the roles and responsibilities questionnaire.

Analysis of the data included exploratory factor analysis and reliability tests to ascertain the validity and reliability of the survey using SPSS. Exploratory factor analysis was conducted with oblimin rotation with the 24 perceived importance items of GTA roles. The eigenvalue-greaterthan-one rule was initially used in combination with a scree test to determine the number of factors that would represent appropriately the perceived importance of GTA roles and responsibilities. We used the eigenvalue-greater-than-one rule ${ }^{[27]}$ and scree test ${ }^{[28]}$, the most frequently and widely used methods, to determine the number of factors. Eigenvalues indicate the total variance accounted by each factor and factors with eigenvalues equal or higher than 1 were retained. The scree test creates a plot of the eigenvalues by ordering each factor by variance and factors above elbow points in the plot (i.e. where sudden decrease in variance is observed) were retained. 


\section{Motivation to Teach}

The Work Tasks Motivation Scale for Teachers (WTMST) ${ }^{[29]}$ was used for this study. The WTMST was designed to assess five constructs in motivation toward six different aspects of teachers' work tasks: class preparation, teaching, evaluation of students, class management, and administrative tasks. We made minor modifications of the original survey items to assess GTAs' motivation to teach. The original scale include five subscales: 1) Intrinsic motivation, 2) Identified motivation, 3) Introjected motivation, 4) Extrinsic motivation, and 5) Amotivation. Out of the five subscales, we utilized three subscales (Intrinsic motivation, extrinsic motivation, and amotivation) relevant for the purpose of the study. We chose this measure to further examine the construct validity of GTA need assessment survey because it is one of the empirically validated measures of teaching beliefs. For each item, such as "I find teaching interesting to do" for intrinsic motivation, "Because the school obliges me to do it" for extrinsic motivation, or "I don't know, sometimes I don't see its purpose" for amotivation, participants chose the rating that most closely corresponded to their reasons for teaching (Strongly disagree, Somewhat disagree, Neutral, Somewhat agree, Strongly agree). We conducted an exploratory factor analysis to evaluate the construct validity of the modified version of the WTMST using GTA self-report data. The result revealed a clear three-factor structure comprising intrinsic motivation, extrinsic motivation, and amotivation. The three factors accounted for $72.21 \%$ of the total variance and showed acceptable reliability coefficients $(\alpha=.79$ for intrinsic motivation, $\alpha=.66$ for extrinsic motivation, $\alpha=.89$ for amotivation). We used the WTMST to evaluate the concurrent validity of our Need Assessment Survey using SPSS to calculate and then to examine correlations between the factors identified in the factor analysis and the teacher motivation measure.

\section{Results}

To assess the construct validity of the measure, an exploratory factor analysis was performed using the principal component analysis. Results yielded four factors with eigenvalues greater than 1, accounting for $54.03 \%$ of the total variance (see Table 1). The scree test further suggested four factors that accounted for $34.95 \%, 8.22 .00 \%, 5.79 \%$, and $5.07 \%$ of the variance, respectively. Most survey items showed factor loadings greater than 0.40 except two items with a loading of 0.36 and 0.34 , respectively and the cross-loadings were not substantial (all were less than 0.30). The first factor consisted of 6 items, reflecting GTAs' perceived importance of their ability to effectively communicate with students and explain course contents clearly, and thus was named 'Clear Communication'. The second factor comprised 5 items reflecting GTAs' role to deal with student behaviors and classroom disruption issues as well as to engage and motivate inattentive/uninterested students, and thus was named 'Student Management'. The third factor contained 5 items that capture GTAs' role to prepare themselves for feedback and assessment. The last factor comprised 7 items that tap into GTAs' responsibilities to become familiar with 
the course objectives and syllabus and holding office hours so we named it as 'Course Management/Policy Knowledge'. Survey items and factor loadings are provided in Table 1.

Table 1: Factor Loadings for Need Assessment Survey for TA Training

\begin{tabular}{|c|c|c|c|c|}
\hline & \multicolumn{4}{|c|}{ Factor } \\
\hline & 1 & 2 & 3 & 4 \\
\hline IMP13: Effectively communicating with students & .81 & & & \\
\hline IMP14: Explaining contents clearly & .77 & & & \\
\hline IMP11: Being familiar with the course materials & .63 & & & \\
\hline IMP6: Engaging the students with the learning material & .62 & & & \\
\hline IMP8: Offering feedback on work to the students & .55 & & & \\
\hline IMP15: Overcoming cultural and language conflicts & .50 & & & \\
\hline IMP22: Maintaining authority over dominating and aggressive students & & .72 & & \\
\hline IMP17: Motivating inattentive/uninterested students & & .70 & & \\
\hline IMP24: Managing students' disruptive classroom behavior & & 67 & & \\
\hline IMP23: Avoiding offending a student while trying to simplify a concept & & .65 & & \\
\hline IMP16: Speaking to the class publicly & & .64 & & \\
\hline IMP21: Facilitating positive team dynamics and discussions & & .57 & & \\
\hline IMP19: Knowing what is expected of the TA & & & .65 & \\
\hline IMP12: Grading student work in a fair and consistent way & & & .57 & \\
\hline IMP18: Knowing answers to student questions (course content) & & & .57 & \\
\hline IMP20: Getting students to think for themselves \& learn to solve problems & & & .55 & \\
\hline IMP10: Treating students with compassion and respect & & & .42 & \\
\hline IMP2: Being familiar with the syllabus & & & & .68 \\
\hline IMP3: Being familiar with course objectives & & & & .65 \\
\hline IMP7: Making your grading rubric available to the students & & & & .59 \\
\hline IMP4: Preventing academic dishonesty & & & & .51 \\
\hline IMP5: Dressing appropriately & & & & .49 \\
\hline IMP9: Holding regular office hours & & & & .36 \\
\hline IMP1: Establishing working relationship with students & & & & .34 \\
\hline Eigenvalue & 8.39 & 1.97 & 1.40 & 1.22 \\
\hline$\%$ of Variance Explained & 34.95 & 8.22 & 5.79 & 5.07 \\
\hline Total Variance Explained & & & $54.03 \%$ & \\
\hline
\end{tabular}


The data provided favourable evidence for internal consistency of the measure. The Chronbach alpha reliability coefficients with the sample of this study for the four factors was 0.81 (Clear Communication), 0.82 (Student Management), 0.77 (Preparation for Feedback and Assessment), and 0.77 (Course Management/Policy Knowledge).

Table 2: Means, Standard Deviations, and Reliabilities of Four Factors

\begin{tabular}{lccc}
\hline & \multicolumn{2}{c}{ Importance } & Reliability \\
\hline & $M$ & $S D$ & Alpha \\
1. Clear Communication & 4.35 & .55 & .81 \\
2. Student Management & 3.84 & .76 & .82 \\
3. Preparation for Feedback and Assessment & 4.42 & .54 & .77 \\
4. Course Management/Policy Knowledge & 4.05 & .59 & .77 \\
\hline
\end{tabular}

Table 2 shows descriptive statistics (means and standard deviations, and reliabilities) of four factors. GTAs placed higher importance on the factors of 'Clear Communication' and 'Course Management/Policy Knowledge' than the other two factors.

We evaluated the concurrent validity of the measure by examining correlations between the four factors and teacher motivation measure which has been empirically validated in the literature. The results revealed that the four factors were positively correlated with GTAs' intrinsic motivation to teach $(r=.18 \sim .32)$ while they showed no relations with either extrinsic motivation or amotivation (see Table 3 ).

Table 3: Correlations between Factors Extracted from Need Assessment Survey and Teacher Motivation

\begin{tabular}{lccccccc}
\hline & 1 & 2 & 3 & 4 & 5 & 6 & 7 \\
\hline 1. Clear Communication & - & & & & & & \\
2. Student Management & $.53^{* *}$ & - & & & & & \\
3. Preparation for Feedback and Assessment & $.66^{* *}$ & $.60^{* *}$ & - & & & & \\
4. Course Management/Policy Knowledge & $.66^{* *}$ & $.52^{* *}$ & $.58^{* *}$ & - & & \\
5. Intrinsic Motivation To Teach & $.22^{* *}$ & $.32^{* *}$ & $.18^{*}$ & $.23^{* *}$ & - & & \\
6. Extrinsic Motivation To Teach & -.02 & -.04 & .01 & .04 & -.15 & - & - \\
7. Amotivation & -.05 & .01 & -.09 & .05 & $-.17^{*}$ & $.47^{* *}$ & - \\
\hline
\end{tabular}

$* p<.05, * * p<.01$

\section{Discussion}

We developed a need assessment survey to measure GTAs' perceived importance of various roles and responsibilities. Our results provide strong evidence that it is a valid and reliable measure. Based on the four-factor structure that an exploratory factor analysis indicated, we 
conceptualized four categories of GTA roles and responsibilities; 1) clear communication, 2) student management, 3) preparation for feedback and assessment, and 4) course management /policy knowledge.

The findings of the current study have theoretical and practical implications for GTA training. Theoretical implication is that GTAs' roles and responsibilities are complex in nature and thus it is important to take multi-dimensional aspects of GTA roles into consideration in the measure of their need for professional development. As shown in Table 2, GTAs placed different levels of importance on each category. GTAs tend to perceive managing and dealing with student behaviors and motivation problems ('Student Management' category) as the area of least importance. This would be consistent with an expectation that such problems should not exist among students enrolled in an engineering discipline or in college generally, especially among the second-year or later students that most GTAs teach. Alternatively or in addition, GTAs may not be inclined to help those they perceive as less motivated or properly engaged succeed, instead thinking that if the students don't take responsibility they should not be pursuing a degree in engineering. If so, this may reflect the GTAs perception of themselves as motivated and interested.

In contrast, GTAs felt highly responsible in areas such as being prepared to perform their primary roles such as grading student work and providing useful feedback ('Preparation for Feedback and Assessment' category), and having an ability to effectively communicate with students to facilitate better learning ('Clear Communication'). These factors may reflect the GTAs experiences as well. They may have received criticism for deficiencies in these areas or be critical of those that taught them and may differ in how they attribute differences in the quality of instruction to specific knowledge, habits, or skills. Providing useful feedback and consistent grading are very desirable characteristics of teachers. Herrington and Nakhleh ${ }^{[17]}$ found that when asked to rank GTA characteristics, GTA and students considered GTA knowledge and skills more important than GTA attitudes toward students, with students ranking consistency in grading higher than GTAs did. Herrington and Nakhleh also found that GTAs and students ranked the GTAs' role in motivating students low.

These results are in direct contrast to the motivation behind GTA training discussed in the Background and Rationale section. This clearly indicates that anyone who wants to develop a training program for the GTAs sampled here must emphasize the role that GTAs play in student development. The training should not only convey that GTAs are responsible for engaging and motivating students, but also provide sound pedagogical tips and exercises on how to engage and motivate students. While this paper focuses on the psychometric properties of the survey, a previous publication ${ }^{[6]}$ offers other more specific examples of how the survey results can be used in developing a GTA training program.

We found that GTAs' ratings of importance had a positive relationship with intrinsic motivation, suggesting that GTAs who enjoy and value what they are doing are highly likely to take more responsibilities in all categories of GTA roles. The positive links between these variables provides not only further evidence of construct validity of the need assessment survey but also provides strong rationale for addressing the four categories of GTA roles in teaching workshops to promote intrinsic interest and value of teaching. 
Although the survey showed favorable psychometric properties, further testing is warranted to confirm that the new measure of GTA's need assessment can be used as a reliable and valid tool across institutions for other institutions to adopt it for guiding their TA training efforts. On a practical stance, this survey helps us design and develop TA training modules that are inclusive and comprehensive to allow for various interest groups to customize their own program of training. For GTAs who may be more extrinsically motivated, informing them of the importance allocated to various characteristics (and by implication the criteria by which they may be evaluated) may serve to motivate them.

\section{References}

1. A. Rugarcia, et al., The Future of Engineering Education. I. A Vision for a New Century. Chemical Engineering Education, 2000. 34(1): p. 16-25.

2. Educating the Engineer of 2020: Adapting Engineering Education to the New Century. 2005, National Academies Press: Washington D.C.

3. P.D. Galloway, The 21st-Century Engineer: A Proposal for Engineering Education Reform. Civil Engineering, November, 2007: p. 46-51.

4. E. Seymour and N. Hewitt, Talking About Leaving: Why Undergraduates Leave the Sciences. 1997: Westview Pr.

5. C. O'Neal, et al., The Impact of Teaching Assistants on Student Retention in the Sciences: Lessons for Ta Training. Journal of College Science Teaching, 2007. 36(5): p. 24-29.

6. Y. Cho, D.P. French, and S. Sohoni, Need Assessment for Graduate Teaching Assistant Training: Identifying Important but under-Prepared Roles, in ASEE Midwest Section Annual Conference. 2010: Lawrence, KS.

7. J. Luft, et al., Growing a Garden without Water: Graduate Teaching Assistants in Introductory Science Laboratories at a Doctoral/Research University. Journal of Research in Science Teaching, 2004. 41(3): p. 211-233.

8. J. Bond-Robinson and R. Rodriques, Catalyzing Graduate Teaching Assistants' Laboratory Teaching through Design Research. Journal of Chemical Education, 2006. 83(2): p. 313.

9. L.R. Prieto, C.A. Yamokoski, and S.A. Myers, Teaching Assistant Training and Supervision: An Examination of Optimal Delivery Modea and Skill Emphases. Journal of Faculty Development, 2007. 21(1): p. 33-43.

10. M. Sundberg and J. Armstrong, The Status of Laboratory Instruction for Introductory Biology in Us Universities. The American Biology Teacher, 1993. 55(3): p. 144-146.

11. N.L. Buerkel-Rothfuss and P.L. Gray, Graduate Teaching Assistant Training in Speech Communication and Noncommunication Departments: A National Survey. Communication Education, 1990. 39: p. $292-307$.

12. J. Luo, L. Bellows, and M. Grady, Classroom Management Issues for Teaching Assistants. Research in Higher Education, 2000. 41: p. 353-383.

13. L.R. Prieto, Teaching Assistants' Preferences for Supervisory Style: Testing a Developmental Model of Gta Supervision. Journal of Graduate Teaching Assistant Development, 1999. 6: p. 1-8.

14. L.R. Prieto and E.M. Altmaier, The Relationship of Prior Training and Previous teaching Experience to Self-Efficacy among Graduate Teaching Assistants. Research In Higher Education, 1994. 35: p. 481-497.

15. M. Anderson, Impostors in the Temple. 1992, New York, NY: Simon \& Schuster.

16. J.M. Civikly and R. Hidalgo, Ta Training as Professional Mentoring, in Preparing Teaching Assistants for Instructional Roles: Supervising Tas in Communication, J.D. Nyquist and D.H. Wulff, Editors. 1992, Speech Communication Association: Annandale, VA. p. 209-213.

17. D.G. Herrington and M.B. Nakhleh, What Defines Effective Chemistry Laboratory Instruction? Teaching Assistant and Student Perspectives. Journal of Chemical Education, 2003. 80(10): p. 1197-1205. 
18. W. Leonard, How College Students Learn Science, in Methods of Effective Teaching and Course Management, E.D. Siebert, M.W. Caprio, and C.M. Lyda, Editors. 1997, Kendal Hunt Publishing: Dubuque, IA.

19. M. Pickering, Teaching the Large Course: Can Ability as a Teaching Assistant Be Predicted? Journal of College Science Teaching, 1988. 18(1): p. 55-56.

20. J. Bond-Robinson and R.A.B. Rodriques, Catalyzing Graduate Teaching Assistants' Laboratory Teaching through Design Research. Journal of Chemical Education, 2006. 83(2): p. 313-323.

21. R.R. Allen and T. Rueter, Teaching Assistant Strategies: An Introduction to College Teaching. 1990, Dubuque, IA: Kendall/Hunt.

22. J.D. Feezel and S.A. Myers, Assessing Graduate Assistant Teacher Communication Concerns. Communication Quarterly, 1997. 45(3): p. 110-124.

23. F.F. Fuller, Concerns of Teachers: A Developmental Characterization. American Educational Research Journal, 1969. 6: p. 207-236.

24. J. Sprague and J.D. Nyquist, in The Challenge of Ta Training in the 1990s: New directions for Teaching and Learning, R.E. Young, Editor. 1989, Jossey-Bass: San Francisco, CA.

25. J.D. Nyquist and D.H. Wulff, Working Effectively with Graduate Assistants. 1996, Thousand Oaks, CA: Sage.

26. Y.F. Mok, Teacher Concerns and Teacher Life Stages. Research in Education, 2005. 73: p. 53-72.

27. H.F. Kaiser and J. Rice, Little Jiffy, Mark Iv. Educational and Psychological Measurement, 1974. 34(1): p. 111-117.

28. R. Cattell, The Scree Test for the Number of Factors. Multivariate Behavioral Research, 1966. 1(2): p. 245276.

29. C. Fernet, et al., The Work Tasks Motivation Scale for Teachers. Journal of Career Assessment, 2008. 16: p. 256-279. 